\title{
Exploring the New Technology for Library Instruction
}

With microcomputers seeming to sprout all over the place like dandelions in May and with other technologies developing faster than our ability to keep up with them, the organizers of this year's Twelfth Annual Workshop on Instruction in Library Use, held at Carleton University, Ottawa, Ontario, on May 18-20, 1983, felt it essential that orientation librarians find out what is going on so as not to be left behind in the dust (or laser tracks) of the accelerating technological express. And since Ottawa is sometimes known as Silicon Valley North, the time and place were both opportune for the theme, "Exploring the New Technology for Library Instruction."

The sessions often reflected the fact that not a lot has been done in the area of library instruction with the new technologies. Discussions were exploratory and the programs demonstrated were experimental in nature. For example, participants saw a program produced on a Vic- 20 microcomputer in BASIC that was intended to orient students to the various parts of a small education library. They also saw one produced on an Apple using Pilot that attempted to teach students the principles of the Library of Congress Subject Headings to show them how to find books by subject. Both presentations revealed that it was possible to write a library instruction program for microcomputers, but neither of them had been used in practice by students.

There were several sessions devoted to videotex, its possibilities, and its potential impact on users. A large project employing videotex which has involved several university and government libraries and commercial firms is the next trial. The iNet (intelligent network) allows for the interfacing of several systems that would otherwise be incompatible with each other. A panel discussion on the iNet trial focused on experiences over the past year and with possible uses of iNet for library orientation and instruction in the future.

Participants also had a chance to hear about computer-assisted instruction in Ontario (outside of library applications), and some had an opportunity to have hands-on experience with CAI. There were also presentations that provided guidelines for evaluating both educational software and hardware.

Another technological development that attracted much attention, both in the exhibit area and in a session, was the videodisc. For the past two years the National Library has been working on a project to explore the possibilities of videodisc in the library. Though the applications for instruction are at the moment impractical because of the expense and technical difficulty of making a disc, the demonstration of various formats on one medium was exciting and held promise for future uses in instructional activities.

While nearly all of these sessions were devoted to new technologies in one way or another, a seminar by an education professor on the various ways of learning generated much discussion and, though not specifically related to computers or technologies, provided insight into fundamental issues that should be taken into account if the new technologies are to have a lasting, positive impact on libraries or, for that matter, on society. The speaker pointed out that people have different ways of learning - rational or deductive, empirical or inductive, and metaphorical or analogical - and that one of these models is usually dominant in most people. The CAI program that will work for one person will not work for another. Unfortunately, he indicated most educational software does not accommodate different ways of learning.

The workshop was well attended by librarians from all over Canada and from parts of the eastern and midwestern United States-a clear indication that interest in the topic is running very high.Janet Carson, Carleton University Library, Ottawa, Ontario.

\section{Is Centralizing Demoralizing?}

ACRL's Education and Behavioral Sciences Section has been studying the recent trend towards merging education and psychology libraries into general collections.

What have been your experiences as you attempt to deal with this new situation? Of particular interest are the following areas: service to users, location and accessibility of materials, staff morale and attitudes, collection development, and staff training.

Please mail your comments and experiences to: Vira C. Hinds, Cohen Library, NAC, City College of New York, 138th Street and Convent Avenue, New York, NY 10031. 